\title{
A Novel Regimen for Perioral Dermatitis by Photodynamic Therapy
}

\author{
Smadar Schreiber \\ Photodynamic Therapy Clinic, Assaf Harofeh Medical Center, Zerifin 70300, Israel
}

\begin{abstract}
Some patients with perioral dermatitis are not relieved despite many medications. These patients confront pain, psychological bother and social/occupational limitations. Being efficient for a wide variety of diseases including infectious inflammative degenerative or tumoral, photodynamic therapy holds a good chance to cure diseases of unknown origin, such as perioral dermatitis. This study presents the results of three women with chronic perioral dermatitis that persisted for years with partial response to medical regimen. They asked for photodynamic treatment to ease off their suffering. The duration of follow-up was up to five years. Following PDT (photodynamic therapy), the patients discontinued medications, redness decreased and eruption disappeared. In these patients, a single PDT treatment provided prolonged relief of perioral dermatitis for up to three years. These data are encouraging but not sufficient. Further study is warranted.
\end{abstract}

Key words: PDT (photodynamic therapy), perioral dermatitis, periorificial dermatitis, face rash.

\section{Introduction}

POD (perioral dermatitis) is also called periorificial dermatitis as it can appear around the mouth nose or eyes. It consists of erythematous papules or pustules. The rash does not reach the vermillion border and a few millimeters around the red roll remain clear.

Two groups of patients are affected: women 16 47 years old, children 7 months to 16 years old with mild predomination to males. POD may last months to years but is usually self limited if handled properly. Differential diagnosis includes: seborrheic dermatitis, acne, rosacea, lip licker's dermatitis, atopic dermatitis, sarcoidosis, histiocytosis, contact dermatitis and sun allergy [1].

It is interesting that POD was introduced in 1960 when topical steroids and fluorinated toothpaste were brought to market. Other factors that may provoke POD are cosmetics including foundation, moistuirizer, night cream and oily lipstick [1].

Atopy in patient or family was described in around

Corresponding author: Smadar Schreiber, M.D., Ph.D., Head of Photodynamic Therapy Clinic, Assaf Harofeh Medical Center, research fields: photodynamic therapy, dermatology, oncology.
$70 \%$ of cases. In some cases, infection was recognized including demodex, fusobacterium and candida. The above are thought to exacerbate or perpetuate POD, but the factor that elicits POD remains unknown in many cases [1].

As a therapy, many clinicians discontinue the usage of steroids and cosmetics and others also discontinue the usage of toothpaste. This "zero use therapy" cures around $90 \%$ of patients within two months $[1,2]$. So medication (Table 1) [3] can be reserved for severe and prolonged POD.

Some patients with POD are not relieved despite the regular medications [1-3]. PDT (photodynamic therapy) was suggested as a novel treatment option for POD, showing high efficacy. More researches are warranted as the study in Ref. [4] was the single dealing with the topic in an internet database search including Pubmed, Medline, Proqeust and Health \& Wellness resource center.

The objective of this study was to evaluate the efficacy of PDT for POD.

\section{Materials and Methods}

This is a review of case notes. Patients that failed 
Table 1 Medication for POD [3] (modified).

\begin{tabular}{ll}
\hline Group & Drugs \\
\hline Systemic antibiotic & Erythromycin, Azythromycin, Tetracycline \\
Topical antibiotic & Erythromycin, Clindamycin, Metronidazole, Sulfacetamide, Benzoyl Peroxide, Antifungal \\
Keratolytic & Adapelene, Azelaic Acid \\
Antiinflammatory & Calcineurin Inhibitor \\
Protective & Barrier cream, topical Vitamin E \\
\hline
\end{tabular}

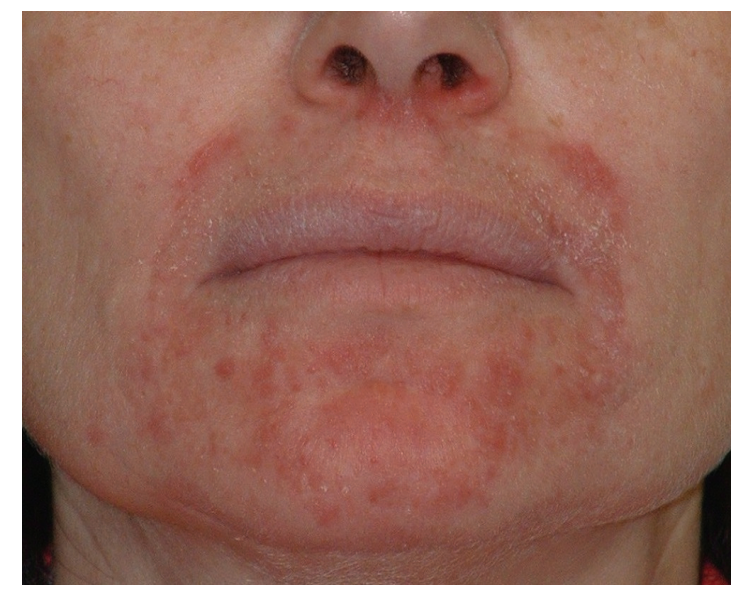

(a)

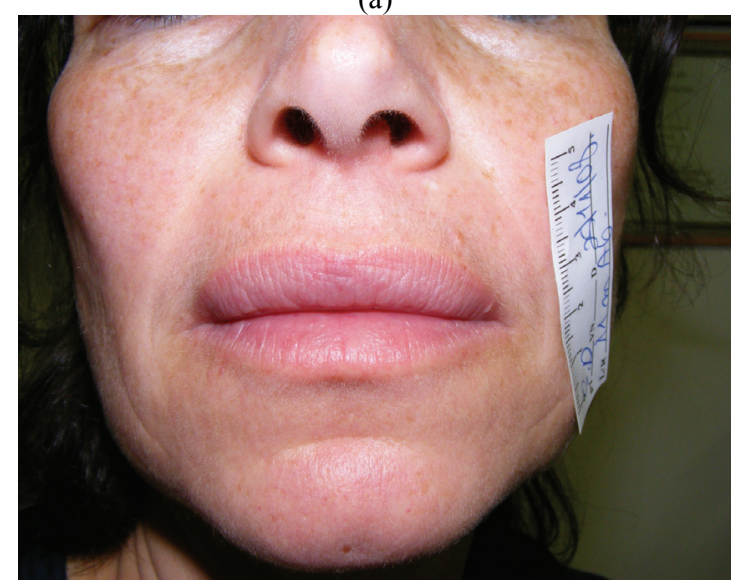

(c)

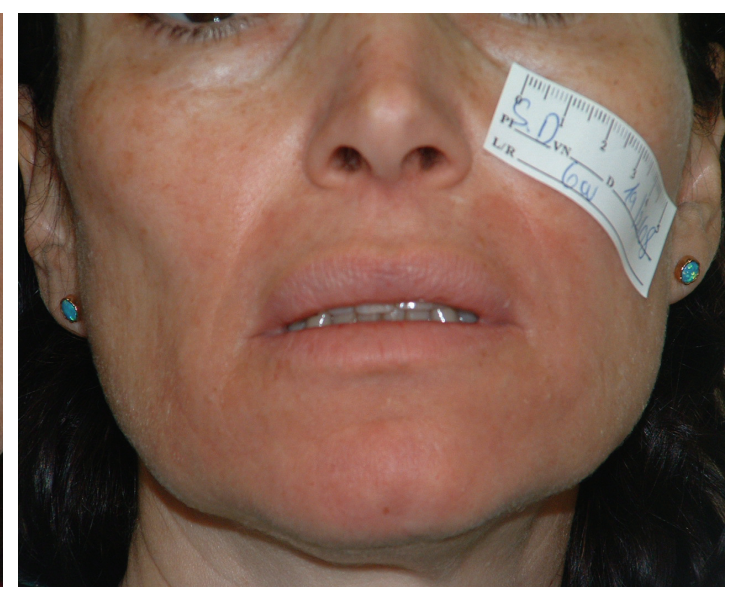

(b)

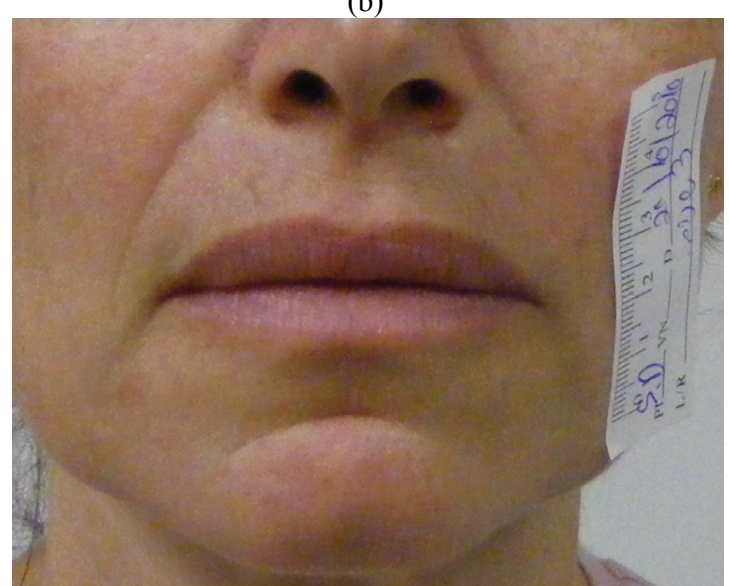

(d)

Fig. 1 Patient 1 follow-up: (a) immediately before a single PDT; (b) at six weeks after a single PDT; (c) at 11 months after a single PDT; (d) at three years after a single PDT.

other treatment regimen sought PDT to try ceasing their suffering although they were told there was no clinical evidence that they benefit. Patients that were pregnant, breast-feeding or treated by steroid during recent week were rejected. ALA 10\% cream was applied for $3 \mathrm{~h}$ followed by $630 \mathrm{~nm}$ illumination of $50 \mathrm{~J} / \mathrm{cm}^{2}$. Patients were photographed before and at follow-ups. Clinical examination and photographs at follow-up were compared with baseline.

\section{Results}

\subsection{Patient 1}

A 48-year-old female with dermatitis around the mouth that persisted a few years (Fig. 1) asked for PDT. The rash was exacerbated by steroid cream and relieved by homeopathic ointment. Her anamnesis included anxiety treated by seroxat, penicillin allergy and benign breast lump. Redness papules and pustules 
were seen around the mouth with minimal scaling. Differential diagnosis of rosacea was less reasonable than POD concerning topology and the lack of flushing. She was cured by a single PDT session. During the three years of follow-up, she remained clear most of time (as shown in Fig. 1c) and was very satisfied. Even when she had a few lesions, they were resolved spontaneously or by using homeopathic ointment: Six weeks after PDT sun exposure was followed by scaling (Fig. 1b), a few times excitement was followed by mild redness, and after three years, a few blemishes showed up (Fig. 1d).

\subsection{Patient 2}

A 22-year-old female came to our clinic in 2007 with dermatitis around the nose perpetuating for three years (Fig. 2a). The rash was relieved by alternate usage of steroid cream (Elocom). Allergy test results were negative. On admission, red papules without

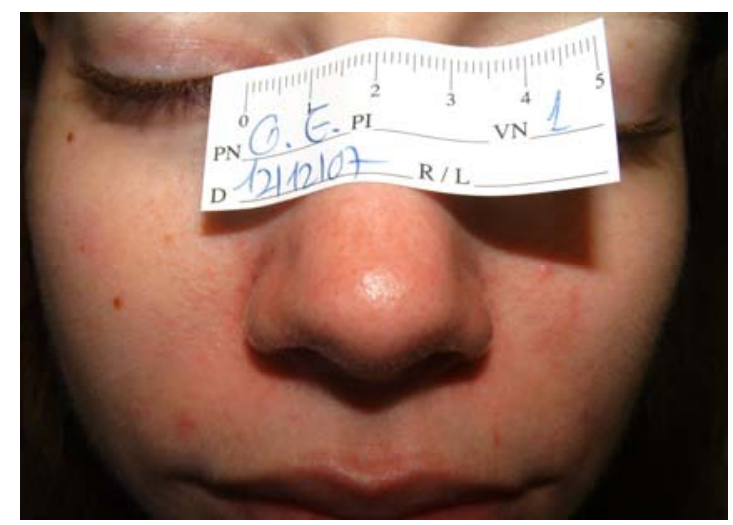

(a)

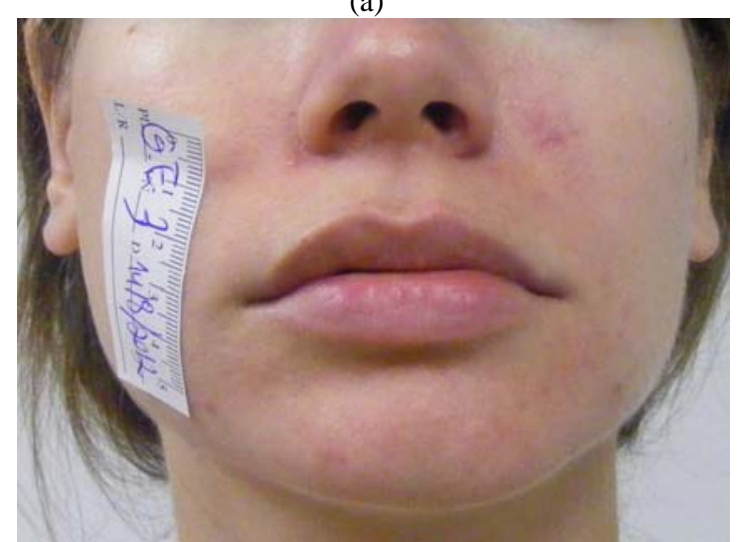

(c) scale were seen around the nose and healing herpes wound of lower lip. Differential diagnosis of rosacea was less reasonable than POD concerning topology and the lack of flushing. She was cured by a single PDT session and remained clear for one year. On 2009 dermatitis, this time around the mouth, flared up consistently for four months (Fig. 2b). Lesions were cured by a single PDT session. She remained clear for another 3.25 years. In 2012, she came back with dermatitis around the mouth and nose (Fig. 2c) of 1-month duration, unresponsive to benzoyl peroxide and partly relieved by antibiotic Fucidin cream and Zindaclin Gel. Lesions were cured by a single PDT (Fig. 2d).

\subsection{Patient 3}

A 50-year-old female with dermatitis around mouth and nose persistent for one year and relieved by steroid cream came to our clinic. According to anamnesis,

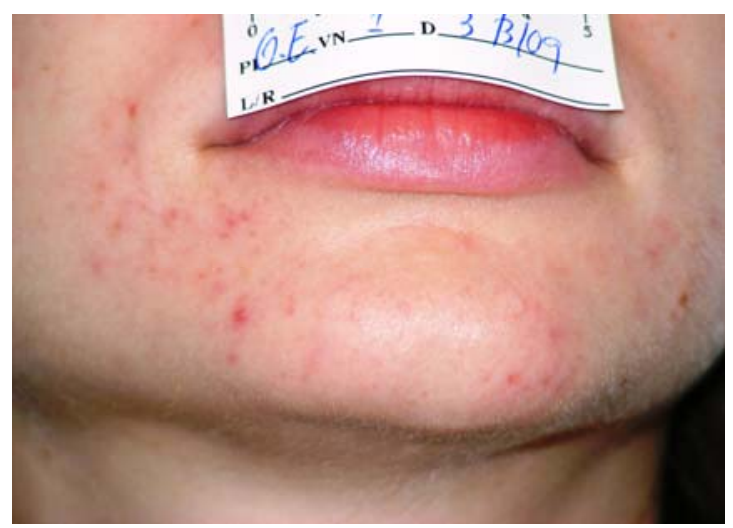

(b)

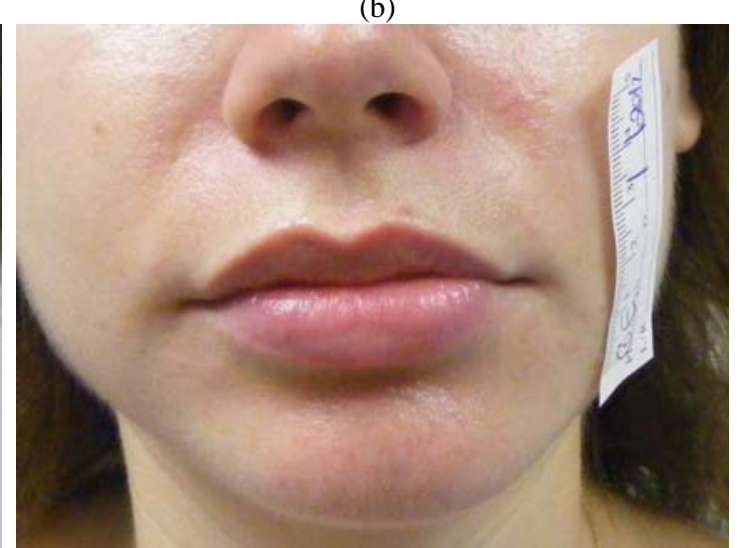

(d)

Fig. 2 Patient 2 follow-up: (a) immediately before PDT sessions in 2007; (b) in 2009; (c) in 2012; (d) at 2.5 months after 2012 session. 


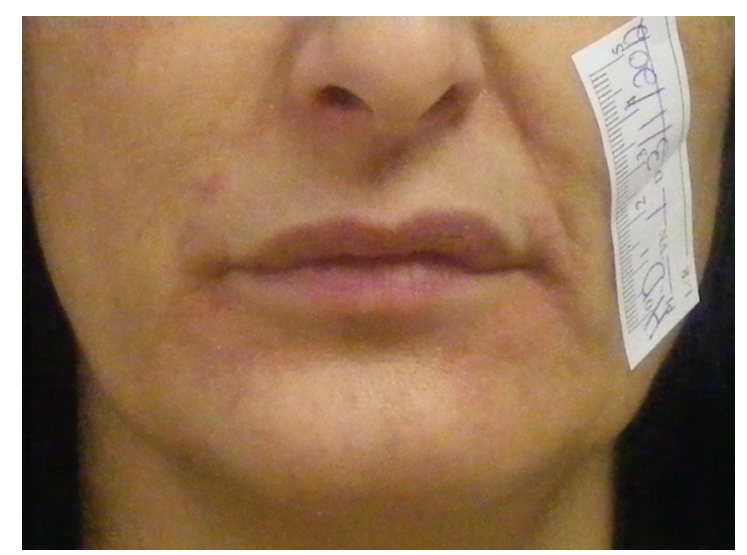

(a)

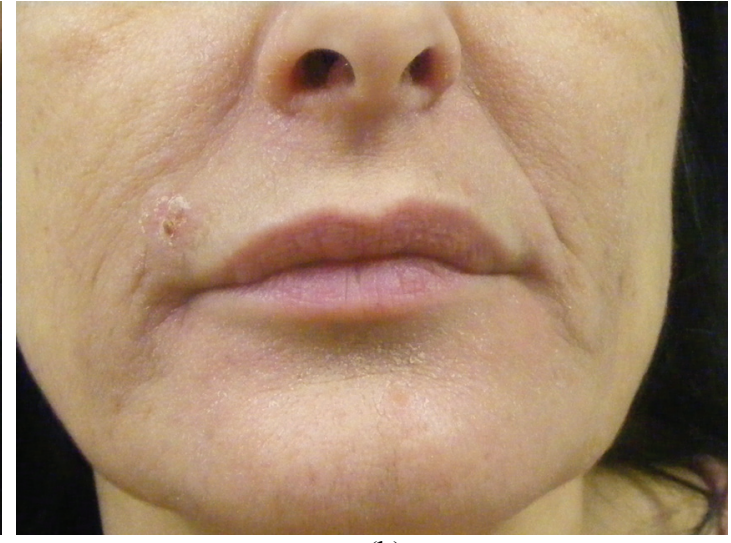

(b)

Fig. 3 Patient 3 follow-up: (a) immediately before PDT; (b) a week after PDT.

five years ago, silicone was injected to lips and nasolabial, eight months ago Botox was injected to forehead and she was treated by antidepressive Tablets (Recital). Red blemishes, papules and scale were seen around the mouth (Fig. 3a). Differential diagnosis included POD, seborrheic dermatitis which seemed less reasonable as lesions were not limited to nose and cheek, or allergic reaction to silicone. She was treated by a single PDT session. One week following, most POD lesions cleared and some crust that can remain two weeks after PDT, were still seen (Fig. 3b). Long-term result is not known as she discontinued follow-up.

\section{Discussion}

The pathogenesis of POD is not completely understood and it may be provoked by multiple factors. Regular treatment regimen extends weeks to months [3] yet some patients remain unresponsive. In this study, POD persisted for years before it was cleared by PDT. This emphasizes the importance of effective treatment options. Patients with prolonged POD confront pain, psychological bother and social/occupational limitations. Being efficient for a wide variety of diseases PDT holds a good chance to cure POD. More specifically PDT can cure inflammatory diseases including acne and rosacea $[5,6]$ that manifest erythematous papules or pustules, such as POD. In addition, POD may be induced or provoked by infection including demodex or fungi that can be alleviated by PDT [5]. The suggested paths of action remain to be elucidated. PDT can cure POD by 1 4 sessions, as shown here and previously. Herein a single PDT was sufficient for long periods. Following PDT and for years, Patient 1 had only minimal flare ups that were spontaneously cured. Patient 2 needed a total of three treatments intermitted 1 3 years and it was shown that PDT can be repeated while keeping its efficacy. A much-longer-than-usual follow-up strengthens the results of this case series. This study is limited by a small number of patients and by a strong bias to selecting those patients with persistent POD that failed other treatment regimen. The previous study of split-face 21-patients is not biased and shows higher efficacy of PDT compared to topical clindamycin [4].

\section{Conclusions}

In these three patients, a single PDT treatment cured POD for up to three years. Following PDT, the faces were cleared and the patients discontinued medications taken for years. PDT for POD showed high efficacy and should be proposed to patients that prefer short-term treatment and those who are unresponsive to medications.

\section{References}

[1] Vanderweil, S. G., and Levin, N. A. 2009. "Perioral Dermatitis: It Is Not Every Rash that Occurs around the Mouth.” Dermatology Nursing 21 (6): 317-53.

[2] Weber, K. 2003. "How I Treat Perioral Dermatitis: 
Non-compliance with the Treatment Guidelines." Dermatology 207 (2): 215.

[3] Caputo, R., and Barbareschi, M. 2007. "Current and Future Treatment Options for Perioral Dermatitis." Expert Review of Dermatology 2 (3): 351-5.

[4] Richey, D. F., and Hopson, B. 2006. "Photodynamic Therapy for Perioral Dermatitis.” J. Drugs Dermatol. 5 (2): 12-6.
[5] Dai, T., Huang, Y., and Hamblin, M. R. 2009. "Photodynamic Therapy for Localized Infections-State of the Art." Photodiagnosis and Photodynamic Therapy 6 (3-4): 170-88.

[6] Bryld, L. E., and Jemec, G. B. E. 2007. "Photodynamic Therapy in a Series of Rosacea Patients.” J. Eur. Acad. Dermatol. Venereol. 21 (9): 1199-202. 